S. Takenaka

Nagoya Math. J.

Vol. 67 (1977), 89-120

\title{
ON PROJECTIVE INVARIANCE OF MULTI-PARAMETER BROWNIAN MOTION
}

\author{
SHIGEO TAKENAKA
}

The multi-parameter Brownian motion introduced by P. Lévy is not only a basic random field but also gives us interesting fine probabilistic structures as well as important properties from the view point of analysis. We shall be interested in investigation of such structures and properties by expressing the Brownian motion in terms of the multiparameter white noise. The expression naturally requires basic tools from analysis, in particular the Radon transform. While there arises the special linear group $S L(n+1, R)$, to which the Radon transform is adapted, and the group plays an important role in observing probabilistic structures of the Brownian motion. To be more interested, we can give some deep insight to unitary representations of $S L(n+1, R)$ through our discussion.

Before we come to our topic, we shall have a quick review of the one dimensional case, emphasizing the following three points:

1) The ordinary Brownian motion $B(t), t \in \boldsymbol{R}$, has an integral representation

$$
B(t)=\int_{0}^{t} \xi_{x} \sqrt{d x} \text {, where }\left\{\xi_{x} \sqrt{d x}\right\} \text { is the white noise on }(\boldsymbol{R}, d x) .
$$

2) Using the white noise $\left\{\xi_{x} \sqrt{d x}\right\}$ we get an isometry $W(\cdot)$ called the Wiener integral from $L^{2}(\boldsymbol{R}, d x)$ into the space of random variables with finite variance $L^{2}(\Omega)$ such that

$$
W(f)=\int_{-\infty}^{\infty} f(x) \xi_{x} \sqrt{d x}
$$

and get the Fock decomposition, where $L^{2}(\Omega)$ can be identified with $\operatorname{Exp} L^{2}(\boldsymbol{R}, d x)$ :

Received July 29, 1976. 


$$
L^{2}(\Omega)=\operatorname{Exp} L^{2}(R, d x) .
$$

3) There exists an important group $F_{1}$ (isomorphic to $S L(2, R)$ ) that expresses the projective invariance of the Brownian motion $B(t)$.

In this paper we shall, by generalizing them, carry out investigations in the multi-parameter case where much fine and interesting structure is found.

Our steps are as follows. Let $(E, \mathbf{B}, \mu)$ be a measure space. In the first place, we consider the Gaussian random measure

$$
\mathscr{X}=\{X(B, \omega) ; B \in \mathbf{B}, \omega \in \Omega\} \quad \text { on } \quad(E, \mathbf{B}, \mu) \quad(\S 1) .
$$

We take a space of step functions on $E$ to be the basic probability space $\Omega(\S 2)$. We assume that a group $G$ acting on $E$ is given and the measure $\mu$ on $E$ is quasi-invariant under the action of $G$. An action of $G$ can be lifted up on the probability space $\Omega$ in such a way that

$$
(g \circ \omega)(x)=\omega(x g), \quad \omega \in \Omega .
$$

This action induces an action on the random measure as

$$
X(B, \omega) \mapsto X^{g}(B, \omega) .
$$

Let $I(\cdot)$ and $I^{g}(\cdot)$ denote the stochastic integrals with respect to the random measure $\mathscr{X}$ and $\mathscr{X}^{g}$, respectively. Then we get the following relation

$$
I^{g}(f(x), \omega)=I\left(\sqrt{\frac{d \mu^{g-1}}{d \mu}} f(x g), \omega\right), \quad f \in L^{2}(E, \mu) \quad \text { (Theorem 3). }
$$

Thus we get a unitary representation of $G$

$$
U_{g} ; f(x) \mapsto \sqrt{\frac{d \mu^{g-1}}{d \mu}} f(x g)
$$

on $L^{2}(E, \mu)$ (but not necessarily be continuous in $g$ ) (§3).

In the next place, identify the $n$-dimensional Euclidean space $\boldsymbol{R}^{n}$ with the $n$-dimensional real projective space $\boldsymbol{P}^{n}$ by using the homogeneous coordinate. Let $M(n)$ be the $n$-dimensional Euclidean motion group, and set

$$
M^{*}(n)=\left\{{ }^{t} g ; g \in M(n)\right\},
$$

where ${ }^{t} g$ denotes the transposed matrix of $g$ (see the matrix form (40) 
of $M(n)$ ). Let $d \Psi$ be an $M^{*}(n)$-invariant measure on $\boldsymbol{P}^{n}$ and let $\left\{\xi_{x} \sqrt{d \bar{\Psi}}\right\}$ be the random measure on the measure space $\left(\boldsymbol{P}^{n}, d \Psi\right)$.

Put

$$
B(a)=\mathrm{p} \cdot \mathrm{v} \cdot\left(\int_{P^{n}\left(\tilde{a}^{*}\right)}-\int_{P^{n}\left(\tilde{\tilde{o}}^{*}\right)}\right) \xi_{x} \sqrt{d \mathrm{Y}(x)},
$$

where $a \in \boldsymbol{R}^{n}$, and $\boldsymbol{P}^{n}\left(\tilde{a}^{*}\right)$ assigns an orientation of $\boldsymbol{P}^{n}-\tilde{a}^{*}$ ( $\tilde{a}^{*}$ is the inversion of $a$ ) which is continued from the origin $O$ of $\boldsymbol{R}^{n}$ through the projection $\pi$. Then $B(a)$ satisfies the following condition 1) and 2):

1) $B(O) \equiv 0$

2) $B(a)-B(b) \simeq N(0,\|a-b\|)$.

That is, $B(a)$ is the Brownian motion with parameter space $\boldsymbol{R}^{n}$ in the sense of P. Lévy [10b], (§4).

In the case of odd dimension $n$, we consider an isometric operator $R$ which links the space $L^{2}\left(\boldsymbol{R}^{n}, d x\right)$ and the space $L^{2}\left(\boldsymbol{P}^{n}, d \varphi\right)$. The operator $R$ is defined by

$$
f(x) \mapsto(R f)(\eta)=\left.\left(\left(\frac{\partial}{\partial p}\right)^{(n-1) / 2} \int_{\langle\xi, x\rangle=p} f(x) d x\right)\right|_{\substack{\|\xi\|=1 \\ \eta=-\frac{1}{p} \xi}},
$$

$x, \xi, \eta \in \boldsymbol{R}^{n}, p \in \boldsymbol{R},(\S 5)$.

Consider the following one-parameter groups of unitary operators acting on $L^{2}\left(\boldsymbol{R}^{n}, d x\right)$ :

1) Shift:

$$
\left(S_{i}(t) f\right)(x)=f\left(x_{1}, \cdots, x_{i-1}, x_{i}+t, x_{i+1}, \cdots, x_{n}\right),
$$

(the flow of the ordinary Brownian motion).

2) Dilation:

$$
\left(D_{i}(t) f\right)(x)=e^{t / 2} f\left(x_{1}, \cdots, x_{i-1}, e^{t} x_{i}, x_{i+1}, \cdots, x_{n}\right) .
$$

Set

$$
\left(J_{i} f\right)(x)=\left(\frac{1}{x_{i}}\right)^{(n+1) / 2} f\left(\frac{x_{1}}{x_{i}}, \cdots, \frac{x_{i-1}}{x_{i}}, \frac{-1}{x_{i}}, \frac{x_{i+1}}{x_{i}}, \cdots, \frac{x_{n}}{x_{i}}\right),
$$

and consider

3) $S_{i j}=J_{i}{ }^{-1} S_{j} J_{i}$, for $i \neq j$,

4) $K_{i}=J_{i}{ }^{-1} S_{i} J_{i}$. 
Then these one-parameter groups induce a quasi-regular representation $T_{g}$ of the group $S L(n+1, R)(\S 6)$.

The measure $d \Psi$ is quasi-invariant under the action of $S L(n+1, R)$, so that we get a unitary representation $U_{g}$ of $S L(n+1, R)(\S 3)$. The relation between these two representations is given by

$$
U_{\hat{g}}=R T_{g} R^{-1},
$$

where $\hat{g}$ is ${ }^{t} g^{-1}$ in the standard matrix form (40), (the main theorem). Thus our approach may be illustrated by the following diagram:

Brownian motion White noise

$$
\downarrow \quad B(a)=\text { p.v. }\left[\int_{P^{n}\left(\tilde{a}^{*}\right)}-\int_{P^{n}\left(\tilde{0}^{*}\right)}\right] \xi_{x} \sqrt{d प(x)} \downarrow
$$

Projective invariance<smiles>[Te][As]=[Te]</smiles>

Fundamental structure group II $S L(n+1, R) \ni g$

P. Lévy has presented in his book "Problèmes concréts d'analyse fonctionnelles" an approach to construct a theory of non-linear functional analysis in which we can find close connections with probability theory. While T. Hida is, in his theory "white noise analysis", realizing the idea of Lévy. The present work, being in line with them, aims at investigations of the multiparameter case. The author hopes that our work is a first step to that proposed approach. In the Hida theory, the properties 1) $\sim 3$ ) of the Brownian motion $B(t)$ play essential roles. Those properties become much complicated, but tell us interesting probabilistic structures in our "multi-parameter white noise analysis". Our main interest is, of course, the multi-parameter theory, however we can reach deeper understanding in the one-parameter case that can be viewed as a special case.

The author expresses his thanks to Professor T. Hida and Professor H. Nomoto for their suggestions and encouragements without which he could not have accomplished this work. 


\section{§1. Random measure}

Let $(E, \mathbf{B}, \mu)$ be a $\sigma$-finite measure space, where $E$ is a set which is to be a parameter space of random phenomena discussed in this paper, $\mathbf{B}$ is a $\sigma$-field of subsets of $E$ and $\mu$ is a $\sigma$-finite measure defined on $\mathbf{B}$. Set $\mathbf{B}_{0}=\{B \in \mathbf{B} ; \mu(B)<\infty\}$. It forms a sub-ring of $\mathbf{B}$. Another measure space $(\Omega, P)$ with $P(\Omega)=1$, that is a probability space, is provided so that we can describe random events. A member of $\Omega$ is denoted by $\omega$ and is called a random parameter.

A Gaussian system is a collection of random variables such that any finite linear combination of the random variables in the system is always Gaussian in distribution. Such a system is viewed as a subset of the Hilbert space $L^{2}(\Omega, P)$ and the closed linear subspace spanned by the system is again a Gaussian system.

We shall start with a particular Gaussian system given by the following:

Definition 1. A Gaussian system $\mathscr{X}=\left\{X(B, \omega) ; B \in \mathbf{B}_{0}, \omega \in \Omega\right\}$ is called a Gaussian random measure if it satisfies the following conditions 1) and 2):

1) $X(B, \omega) \simeq N(0, \mu(B))$, for any $B \in \mathbf{B}_{0}$, that is, $X(B, \omega)$ is subject to the Gaussian law $N(0, \mu(B))$ which has mean zero and variance $\mu(B)$.

2) $X\left(B_{1} \cup B_{2}, \omega\right)=X\left(B_{1}, \omega\right)+X\left(B_{2}, \omega\right)$ with probability one, for any disjoint pair $B_{1}, B_{2}$ in $\mathbf{B}_{0}$.

The conditions 1) and 2) imply that $X\left(B_{1}, \omega\right)$ and $X\left(B_{2}, \omega\right)$ in $\mathscr{X}$ with $B_{1} \cap B_{2}=\emptyset$ are mutually independent and that $X\left(B_{k}, \omega\right)$ converges to $X(B, \omega)$ for almost all $\omega$, if $B_{1} \subset B_{2} \subset \cdots, \cup_{k}^{\infty} B_{k}=B \in \mathbf{B}_{0}$.

Set

$\mathscr{L}_{0}=\left\{h=\sum_{i=1}^{n} a_{i} \chi_{B_{i}} ;\left\{B_{i}\right\}\right.$ is a mutually disjoint family of $\left.\mathbf{B}_{0}, n \in N, a_{i} \in \boldsymbol{R}\right\}$,

where $\chi_{B_{i}}$ denotes the caracteristic function of the set $B_{i}$.

Then $\mathscr{L}_{0}$ is a dense subspace of the Hilbert space $L^{2}(E, \mu)$ consisting of square summable real functions. With each element $h=\sum a_{i} \chi_{B_{i}}$ of $\mathscr{L}_{0}$ we associate a random variable

$$
I(h, \omega)=\sum a_{i} X\left(B_{i}, \omega\right) .
$$

Then $I(h, \omega)$ is a Gaussian random variable with mean zero and variance 


$$
\mathrm{E}(I(h, \omega))^{2}=\sum a_{i}^{2} \mu\left(B_{i}\right)=\|h\|^{2} .
$$

Therefore $I(\cdot)$ gives an isometric linear map from $\mathscr{L}_{0}$ to $L^{2}(\Omega, P)$, the space of random variables with finite variance. Since $\mathscr{L}_{0}$ is dense in $L^{2}(E, \mu)$, we can extend the map $I(\cdot)$ to an isometry from $L^{2}(E, \mu)$ into $L^{2}(\Omega, P)$. We denote $I(f, \omega)$ by $\int_{E} f(\alpha) d X(\alpha, \omega)$ and call it the stochastic integral of $f \in L^{2}(E, \mu)$ with respect to the random measure $\mathscr{X}$. It is more suggestive to use the notation

$$
d X(\alpha, \omega)=\xi_{\alpha} \sqrt{d \mu(\alpha)}
$$

with this we can regard $\left\{\xi_{\alpha} ; \alpha \in E\right\}$ as a system of random variables which is independent at every point where each $\xi_{\alpha}$ is subject to the same probability law $N(0,1)$.

Let $\mu^{\prime}$ be a measure which is absolutely continuous with respect to the measure $\mu$. Denote by $\rho$ the Radon-Nikodym derivative $d \mu^{\prime} / d \mu$. Then $\sqrt{\rho} \chi_{B}$ is an element of $L^{2}(E, \mu)$ for $B \in \mathbf{B}_{0}^{\prime}=\left\{B \in \mathbf{B} ; \mu^{\prime}(B)<\infty\right\}$. Put

$$
X^{\prime}(B, \omega)=I\left(\sqrt{\rho} \chi_{B}, \omega\right)
$$

Then $X^{\prime}(B, \omega)$ is a Gaussian random variables with variance

$$
\mathrm{E}\left(X^{\prime}(B, \omega)\right)^{2}=\left\|\sqrt{ } \rho \chi_{B}\right\|^{2}=\int_{B} \frac{d \mu^{\prime}}{d \mu} d \mu=\mu^{\prime}(B),
$$

and for any disjoint pair $B_{1}$ and $B_{2}$ of $\mathbf{B}_{0}^{\prime}$ it holds that

$$
\begin{aligned}
X^{\prime}\left(B_{1} \cup B_{2}\right) & =I\left(\sqrt{\rho}\left(\chi_{B_{1}}+\chi_{B_{2}}\right)\right)=I\left(\sqrt{\rho} \chi_{B_{1}}\right)+I\left(\sqrt{\rho} \chi_{B_{2}}\right) \\
& =X^{\prime}\left(B_{1}\right)+X^{\prime}\left(B_{2}\right) .
\end{aligned}
$$

Therefore the system $\mathscr{X}^{\prime}=\left\{X^{\prime}(B, \omega) ; B \in \mathbf{B}_{0}^{\prime}\right\}$ is a Gaussian random measure on $\left(E, \mathbf{B}, \mu^{\prime}\right)$.

Suppose that a group $G$ acts on $E$ and that the measure $\mu$ is invariant under the action of $G$. In this case we call the Gaussian random measure $\mathscr{X}$ on $E$ a $G$-white noise (or simply a white noise). If $\mu$ is quasiinvariant we call $\mathscr{X}$ a G-quasi-white noise.

\section{§2. Construction of random measure}

In this section we will construct a Gaussian random measure $\mathscr{X}$ on $E$ as a function on a certain function space on $E$. 


\section{The case where $\mu(E)$ is finite}

step $1^{\circ}$. Let $\mathscr{N}$ be the totality of non-null finite Borel partitions of $E$, that is,

$$
\mathscr{N}=\left\{\begin{array}{cl}
\nu=\left\{B_{1}, \cdots, B_{n}\right\}, B_{i} \in \mathbf{B}, B_{i} \text { 's are mutually disjoint, } \\
\nu ; & E=\cup B_{i}, \mu\left(B_{i}\right)>0, n \text { is a positive integer }
\end{array}\right\},
$$

and set $|\nu|=$ number of elements of B-sets of $\nu$.

The notation $\nu\left\langle\nu^{\prime}\right.$ means that $\nu^{\prime}$ is a refinement of $\nu$. With this notation we can consider a directed set $\{\mathscr{N},<\}$. Let

$$
\Omega^{\nu}=\left\{\sum_{i} a_{i} \chi_{B_{i}} ; B_{i} \in \mathbf{B}, a_{i} \in \mathbf{R}\right\}
$$

denote the set of $\nu$-step functions. In an obvious manner we identify $\Omega^{\nu}$ with $R^{|\nu|}$ and we introduce the topological $\sigma$-field $\mathbf{F}^{\nu}$ together with a probability $P^{|\nu|}$ which is subject to the $|\nu|$-dimensional normal distribution $(N(0,1))^{|\nu|}$.

For any pair of partitions $\left(\nu, \nu^{\prime}\right)$ with $\nu<\nu^{\prime}$, we define a projection $\pi_{\nu, \nu^{\prime}}$ from the space $\Omega^{\nu^{\prime}}$ onto $\Omega^{\nu}$ :

$$
\sum a_{i j} \chi_{B_{i j}} \mapsto \pi_{\nu, \nu^{\prime}}\left(\sum a_{i j} \chi_{B_{i j}}\right)=\sum_{i}\left(\sum_{j} a_{i j} \sqrt{\frac{\mu\left(B_{i j}\right)}{\mu\left(B_{i}\right)}}\right) \chi_{B_{i}},
$$

where $\nu^{\prime}=\left\{B_{i j}\right\}, \nu=\left\{B_{i}\right\}$ such that $B_{i}=\bigcup_{j} B_{i j}$.

LEMMA 1. The system $\left\{\left(\Omega^{\nu}, \mathbf{F}^{\nu}, P^{\nu}\right), \pi_{\nu, \nu^{\nu}}, \mathscr{N}\right\}$ is a projective system of probability spaces.

Proof. 1) Suppose that $\nu<\nu^{\prime}<\nu^{\prime \prime}$ where $\nu$ and $\nu^{\prime}$ are as above and where $\nu^{\prime \prime}=\left\{B_{i j k}\right\}, B_{i j}=\cup_{k} B_{i j k}$. Then for any element $\omega^{\nu^{\prime \prime}}=\sum a_{i j k} \chi_{B_{i j k}}$ we have

$$
\pi_{\nu, \nu^{\prime \prime}}\left(\omega^{\nu^{\prime \prime}}\right)=\sum_{i} \frac{1}{\sqrt{\mu\left(B_{i}\right)}}\left(\sum_{j, k} a_{i j k} \sqrt{\mu\left(B_{i j k}\right)}\right) \chi_{B_{i j k}}
$$

and

$$
\begin{aligned}
\left(\pi_{\nu, \nu^{\prime}} \circ \pi_{\nu^{\prime}, \nu^{\prime \prime}}\right)\left(\omega^{\nu^{\prime \prime}}\right) & =\pi_{\nu, \nu^{\prime}}\left(\sum_{i} \sum_{j} \frac{1}{\sqrt{\mu\left(B_{i j}\right)}}\left(\sum_{k} a_{i j k} \sqrt{\mu\left(B_{i j k}\right)} \chi_{B_{i j}}\right)\right) \\
& =\sum_{i} \frac{1}{\sqrt{\mu\left(B_{i}\right)}} \sum_{j} \sqrt{\frac{\mu\left(B_{i j}\right)}{\mu\left(B_{i j}\right)}}\left(\sum_{k} a_{i j k} \sqrt{\mu\left(B_{i j k}\right)}\right) \chi_{B_{i}} .
\end{aligned}
$$


These show the following relation:

$$
\pi_{\nu, \nu^{\prime \prime}}\left(\omega^{\nu^{\prime \prime}}\right)=\left(\pi_{\nu, \nu^{\prime}} \circ \pi_{\nu^{\prime}, \nu^{\prime \prime}}\right)\left(\omega^{\nu^{\prime \prime}}\right) \text {. }
$$

2) Set $A^{\nu}=\left\{\omega^{\nu}=\sum a_{i} \chi_{B_{i}} ; a_{i} \in V_{i}\right\}$, where $V_{i}$ 's are Borel subset of $\boldsymbol{R}$. By the definition of $\left(\Omega^{\nu}, P^{\nu}\right),\left\{a_{i}\right\}$ and $\left\{a_{i j}\right\}$ are systems of mutually independent random variables with the same probability law $N(0,1)$. Then

$P^{\nu}\left(A^{\nu}\right)=\prod^{|\nu|} Q\left(a_{i} \in V_{i}\right)$, where $Q$ is the 1-dimensional distribution $N(0,1)$. On the other hand we see that

$$
\begin{aligned}
& P^{\nu^{\prime}}\left(\pi_{\nu, \nu^{\prime}}^{-1}\left(A^{\nu}\right)\right)=\prod^{|\nu|} P^{\left|\nu_{i}^{\prime}\right|}\left(\sum_{j}^{\left|\nu^{\prime}\right|} \sqrt{\frac{\mu\left(B_{i j}\right)}{\mu\left(B_{i}\right)}} a_{i j} \in V_{i}\right), \quad \text { and } \\
& P^{\left|\nu^{\prime}\right|}\left(\sum_{j}^{\left|\nu_{i}^{\prime}\right|} \sqrt{\frac{\mu\left(B_{i j}\right)}{\mu\left(B_{i}\right)}} a_{i j} \in V_{i}\right)=Q\left(a_{i} \in V_{i}\right),
\end{aligned}
$$

where $B_{i}=\bigcup_{j}^{\left|\nu_{i}^{\prime}\right|} B_{i j}$.

Therefore

$$
P^{\nu}\left(A^{\nu}\right)=P^{\nu^{\prime}}\left(\pi_{\nu, \nu^{\prime}}^{-1}\left(A^{\nu}\right)\right)
$$

Thus we have

$P^{\nu}\left(B^{\nu}\right)=P^{\nu^{\prime}}\left(\pi_{\nu, \nu^{\prime}}^{-1}\left(B^{\nu}\right)\right)$, for any Borel subset $B^{\nu}$ of $\Omega^{\nu}$.

q.e.d.

By Bochner's extension theorem (S. Bochner [1]) the projective system $\left\{\left(\Omega^{\nu}, P^{\nu}\right), \pi_{\nu, \nu^{\nu}}\right\}$ defines a projective limit probability space. We denote the projective limit by $(\Omega, \mathbf{F}, P)$ and let

$$
\pi_{\nu} ;(\Omega, P) \mapsto\left(\Omega^{\nu}, P^{\nu}\right)
$$

be the canonical projection.

step $2^{\circ}$. Consider a random variable $X^{\nu}$ defined on $\Omega^{\nu}$ given by

$$
\sum a_{i} \chi_{B_{i}} \mapsto X^{\nu}\left(\sum a_{i} \chi_{B_{i}}\right)=\sum \sqrt{\mu\left(B_{i}\right)} a_{i} .
$$

Furthermore let $\pi^{\nu, \nu^{\prime}}$ be the dual map of $\pi_{\nu, \nu^{\prime}}$;

$$
\pi^{\nu, \nu^{\prime}}\left(X^{\nu}\right)=X^{\nu} \circ \pi_{\nu, \nu}
$$

Then, we have the following lemma.

LEMMA 2. The relation

$$
\pi^{\nu, \nu^{\prime}}\left(X^{\nu}\right)=X^{\nu^{\prime}},
$$


holds. That is, $\left\{X^{\nu}, \pi^{\nu, \nu^{\prime}}\right\}$ is an inductive system of random variables defined on the projective system $\left\{\left(\Omega^{\nu}, \mathbf{F}^{\nu}, P^{\nu}\right), \pi_{\nu, \nu^{\prime}}\right\}$.

Proof. Let $\omega^{\nu^{\prime}}=\sum a_{i j} \chi_{B_{i j}}$ be an element of $\Omega^{\nu}$. Then

$$
X^{\nu}\left(\sum a_{i j} \chi_{B_{i j}}\right)=\sum_{i, j} \sqrt{\mu\left(B_{i j}\right)} a_{i j}
$$

On the other hand

$$
\begin{aligned}
X^{\nu}\left(\pi_{\nu, \nu^{\prime}}\left(\sum a_{i j} \chi_{B_{i j}}\right)\right) & =X^{\nu}\left(\sum_{i}\left(\sum_{j} \sqrt{\frac{\mu\left(B_{i j}\right)}{\mu\left(B_{i}\right)}} a_{i j}\right) \chi_{B_{i}}\right) \\
& =\sum_{i} \sqrt{\mu\left(B_{i}\right)}\left(\sum_{j} \sqrt{\frac{\mu\left(B_{i j}\right)}{\mu\left(B_{i}\right)}} a_{i j}\right),
\end{aligned}
$$

and we have

$$
X^{\nu^{\prime}}\left(\omega^{\nu^{\prime}}\right)=X^{\nu}\left(\pi_{\nu, \nu^{\prime}}\left(\omega^{\nu^{\prime}}\right)\right) .
$$

Denote by $\tilde{X}$ the inductive limit random variable of the inductive system of random variables $\left\{X^{\nu}, \pi^{\nu, \nu^{\prime}}\right\}$ defined on $\Omega$.

step $3^{\circ}$. For any element $B$ of $\mathbf{B}$, denote by $\operatorname{Proj}_{B}$ the projection

$$
\omega^{\nu}=\sum a_{i} \chi_{B_{i}} \mapsto \operatorname{Proj}_{B}\left(\omega^{\nu}\right)=\sum a_{i} \chi_{B_{i} \cap B}
$$

for any $\nu>\left\{B, B^{c}\right\}$, and set

$$
X(B, \omega)=\operatorname{Proj}_{B} \tilde{X}(\omega)=\lim _{\nu>\left\{B, B^{c}\right\}} X^{\nu}\left(\operatorname{Proj}_{B}\left(\pi_{\nu}(\omega)\right) .\right.
$$

THeOREM 1. The system of random variables $\{X(B, \omega) ; B \in \mathbf{B}\}$ is a Gaussian random measure defined on $(E, \mathbf{B}, \mu)$.

Proof. 1) Let $\nu=\left\{B, B^{c}\right\}$ be an element of $\mathscr{N}$. Then,

$$
X(B, \omega)=\operatorname{Proj}_{B} \tilde{X}(\omega)=X^{\nu}\left(\operatorname{Proj}_{B}\left(\omega^{\nu}\right)\right)=c_{B} \sqrt{\mu(B)},
$$

where $c_{B}$ is a standard Gaussian random variables. That is

$$
X(B, \omega) \simeq N(0, \mu(B)) .
$$

2) Take any disjoint pair $\left(B_{1}, B_{2}\right)$, and set $\nu=\left\{B_{1}, B_{2},\left(B_{1} \cup B_{2}\right)^{c}\right\}$. Then by the definition (10), we have

$$
\begin{aligned}
X\left(B_{1} \cup B_{2}, \omega\right) & =X^{\nu}\left(\operatorname{Proj}_{B_{1} \cup B_{2}}\left(\omega^{\nu}\right)\right)=X^{\nu}\left(\operatorname{Proj}_{B_{1}}\left(\omega^{\nu}\right)\right)+X^{\nu}\left(\operatorname{Proj}_{B_{2}}\left(\omega^{\nu}\right)\right) \\
& =X\left(B_{1}, \omega\right)+X\left(B_{2}, \omega\right) .
\end{aligned}
$$




\section{The case where $\mu(E)$ is infinite}

Take a sequence $\left\{E_{i} ; i=1,2, \cdots\right\}$ of subspaces of $E$ such that $E_{i} \subset E_{i+1}, \mu\left(E_{i}\right)<\infty$ and $\bigcup_{i} E_{i}=E$. Put $\mathbf{B}_{i}=\left.\mathbf{B}\right|_{E_{i}}$ and $\mu_{i}=\left.\mu\right|_{E_{i}}$. For each finite measure space $\left(E_{i}, \mathbf{B}_{i}, \mu_{i}\right)$, we find the inductive limit random variable $\tilde{X}^{i}$ on $\Omega^{i}$ constructed in the steps $1^{\circ}$ and $2^{\circ}$ in I. Define the projection $\tilde{\pi}_{i, j}, j<i$, which maps space $\Omega^{j}$ onto $\Omega^{i}$ such that

$$
\omega^{j} \mapsto \tilde{\pi}_{i, j}\left(\omega^{j}\right)=\omega^{j} \circ \chi_{E_{i}} .
$$

Then we get

THEOREM 2. The collection $\left\{\left(\Omega^{j}, P^{j}\right), \tilde{\pi}_{i, j}\right\}$ is a projective system determining a probability space $(\Omega, P)$ as its projective limit. And the system $\left\{\tilde{X}^{i}, \tilde{\pi}^{i, j}\right\}$ is an inductive system of random variables which defines a random variable $\tilde{X}$ as its inductive limit. The system

$$
\mathscr{X}=\left\{X(B, \omega)=\operatorname{Proj}_{B} \tilde{X}(\omega) ; B \in \mathbf{B}_{0}\right\}
$$

is a Gaussian random measure on $(E, \mathbf{B}, \mu)$.

The proof is obvious, since $\left\{\left(\Omega^{j}, P^{j}\right), \pi_{i, j}\right\}$ is a sequencially maximal topological projective system of probability spaces.

In general, given a stochastic integral $I(\cdot)$ we can get the Fock decomposition of the Hilbert space $L^{2}(\Omega, P)$, the space of random variables with finite variance:

$$
L^{2}(\Omega, P)=\operatorname{Exp} L^{2}(E, \mu)
$$

(N. Wiener [14]). For example, the inclusion map of the element $f \otimes g$ of $L^{2}(E, \mu) \hat{\otimes} L^{2}(E, \mu)$ into $L^{2}(\Omega, P)$ is defined as

$$
I(f \otimes g, \omega)=I(f, \omega) \times I(g, \omega)-\langle f, g\rangle,
$$

and its norm is

$$
E(I(f \otimes g, \omega))^{2}=\|f\|^{2} \cdot\|g\|^{2}, \quad\|\cdot\|^{2}=\langle\cdot, \cdot\rangle .
$$

The inclusion $L^{2}(\Omega, P) \subset \operatorname{Exp} L^{2}(E, \mu)$ can be proved by using the fact that $\left\{\exp \left(I(f, \omega)-\frac{1}{2}\|f\|^{2}\right) ; f \in L^{2}(E, \mu)\right\}$ is dense in $L^{2}(\Omega, P)$ (T. Hida [5c] and N. Kôno [8]).

\section{§. White noise and group action on it}

Let $\mathscr{X}$ be a quasi-white noise on a measure space $(E, \mathbf{B}, \mu)$ on which 
a continuous group $G$ acts, and let $I(\cdot)$ be the stochastic integral with respect to the measure $\mathscr{X}$.

Since the probability space $\Omega$, on which the random measure $\mathscr{X}$ is defined, is a space of functions on $E$, we can define the action $G$ on $\Omega$ in such a way that

$$
g \circ \omega=\left(g \circ \omega^{\nu}\right), \quad \text { for } \omega=\left(\omega^{\nu}=\sum a_{i} \chi_{B_{i}} ; \nu \in \mathscr{N}\right)
$$

where $g \circ \omega^{\nu}=\sum a_{i} \chi_{B_{i} g-1}$.

Put

$$
\tilde{X}^{g}(B, \omega)=X(B, g \circ \omega), \quad \text { for } B \text { in } \mathbf{B}_{0} .
$$

Then we can easily obtain the following proposition.

Proposition 1. The system $\tilde{\mathscr{X}}^{g}=\left\{\tilde{X}^{g}(B, \omega)\right\}$ is a Gaussian random measure on $\left(E, \mu^{g}\right)$, where $\mu^{g}(B)=\mu\left(B g^{-1}\right)$ for $B \in \mathbf{B}$.

Let $\tilde{I}^{g}(\cdot)$ denote the stochastic integral with respect to the random measure $\overline{\mathscr{X}}^{g}$. Then,

LEMMA 3.

$$
\tilde{I}^{g}(f(x), \omega)=I(f(x g), \omega),
$$

where $f \in L^{2}\left(E, \mu^{g}\right)$.

Proof. It is sufficient to prove the statement in the case of a step function $\phi(x)=\sum a_{i} \chi_{B_{i}}(x)$.

$$
\begin{aligned}
\tilde{I}^{g}\left(\sum a_{i} \chi_{B_{i}}, \omega\right) & =\sum a_{i} \tilde{I}^{g}\left(\chi_{B_{i}}, \omega\right)=\sum a_{i} \tilde{X}^{g}\left(B_{i}, \omega\right)=\sum a_{i} X\left(B_{i}, g \circ \omega\right) \\
& =\sum a_{i} X\left(B_{i} g^{-1}, \omega\right)=I\left(\sum a_{i} \chi_{B_{i} g^{-1}}, \omega\right) .
\end{aligned}
$$

That is,

$$
\tilde{I}^{g}(\phi(x), \omega)=I(\phi(x g), \omega) . \quad \text { q.e.d. }
$$

On account of (19) the system of random variables $\mathscr{X}^{g}=\left\{X^{g}(B, \omega) ; B \in \mathbf{B}_{0}\right\}$ defined by

$$
X^{g}(B, \omega)=\tilde{I}^{g}\left(\sqrt{\frac{d \mu}{d \mu^{g}}} \chi_{B}, \omega\right)
$$

is a Gaussian random measure on $(E, \mathbf{B}, \omega)$.

Let us apply this map to the stochastic integral and the Fock decomposi- 
tion.

Set $I^{g}(f, \omega)$ be the integration of $f \in L^{2}(E, \mu)$ with respect to the random measure $\mathscr{X}^{g}$. Then we have Theorem 3 .

THEOREM 3. Let $f$ be an element of $L^{2}(E, \mu)$, then

$$
I^{g}(f(x), \omega)=I\left(\sqrt{\frac{d \mu^{g-1}}{d \mu}}(x) f(x g), \omega\right) .
$$

Proof. It is sufficient to prove the relation (21) for a step function $\phi(x)=\sum a_{i} \chi_{B_{i}}$.

$$
\begin{aligned}
I^{g}\left(\sum a_{i} \chi_{B_{i}}, \omega\right) & =\sum a_{i} X^{g}\left(B_{i}, \omega\right)=\sum a_{i} \tilde{I}^{g}\left(\sqrt{\frac{d \mu}{d \mu^{g}}} \chi_{B_{i}}, \omega\right) \\
& =\sum a_{i} I^{g}\left(\left(\sqrt{\frac{d \mu}{d \mu^{g}}} \chi_{B_{i}}\right)(x g), \omega\right)=\sum a_{i} I^{g}\left(\sqrt{\frac{d \mu^{g-1}}{d \mu}} \chi_{B_{i} g-1}, \omega\right) \\
& =I\left(\sqrt{\frac{d \mu^{g-1}}{d \mu}}(x) \phi(x g), \omega\right) .
\end{aligned}
$$

We will write the equation (21) in the following convenient form:

$$
\int_{E} f(x) \xi_{x}^{g} \sqrt{d \mu(x)}=\int_{E} \sqrt{\frac{d \mu^{g-1}}{d \mu}}(x) f(x g) \xi_{x} \sqrt{d \mu(x)} .
$$

The unitary operators defined on $L^{2}(E, \mu)$

$$
\left(U_{g} f\right)=\sqrt{\frac{d \mu^{g-1}}{d \mu}}(x) f(x g), \quad g \in G,
$$

is called a quasi-regular unitary representation of $G$ on $L^{2}(E)$, (but this representation is not necessarily continuous).

This representation $U_{g}$ is lifted up to the unitary representation $U_{g}$ of $G$ on $L^{2}(\Omega, P)$

$$
\tilde{U}_{g} ; I(f, \omega) \mapsto I^{g}(f, \omega) .
$$

We express this fact by the formula

$$
\tilde{U}_{g}=\operatorname{Exp} U_{g}
$$

\section{§4. White noise and Brownian motion}

It is well known that the ordinary Brownian motion $B(t)$, where $t$ runs over the whole real line, can be represented by an integration of 
the characteristic function $\chi_{[0, t)}$ with respect to the white noise $\left\{\xi_{x} \sqrt{d x}\right\}$ on $\left(\boldsymbol{R}^{1}, d x\right)$ :

$$
B(t)=\int_{0}^{t} \xi_{x} \sqrt{d x} .
$$

We will, in this section, extend the relation (25) to the $\boldsymbol{R}^{n}$-parameter case.

I. Let $(M, d)$ be a metric space with metric $d(\cdot)$ and fix a point $O$, call it the origin, in $M$.

Definition 2 (P. Lévy [10b]). A Gaussian system $\{B(m, \omega) ; m \in M$, $\omega \in \Omega\}$ is called a Brownian motion with parameter space $(M, d)$, if the conditions 1) and 2) hold:

$$
\begin{aligned}
B(O, \omega) & =0, \\
B(m, \omega)-B(n, \omega) & \simeq N(0, d(m, n)) .
\end{aligned}
$$

By the definition the covariance function is given by

$$
\begin{aligned}
E(B(m) B(n)) & =\frac{1}{2}\left\{\mathrm{E}|B(m)|^{2}+\mathrm{E}|B(n)|^{2}-\mathrm{E}|B(m)-B(n)|^{2}\right\} \\
& =\frac{1}{2}\{d(m, 0)+d(n, 0)-d(m, n)\} .
\end{aligned}
$$

Existence of such a Gaussian system is guaranteed by the following theorem.

THEOREM 4 (Schönberg-Schwartz c.f. P. Lévy [10b]). A necessary and sufficient condition for the existence of the Brownian motion with parameter space $(M, d)$ is that for any $n \in N$ and for any $\tilde{\tilde{x}}$ of $n$-tuple $\tilde{\tilde{x}}=\left(x_{1}, x_{2}, \cdots, x_{n}\right)$ of elements of $M$, the function $Q(n, \tilde{\tilde{x}})$

$$
Q(n, \tilde{\tilde{x}})\left(\alpha_{1}, \cdots, \alpha_{n}\right)=\sum_{i, j}^{n}\left[d\left(x_{i}, O\right)+d\left(x_{j}, O\right)-d\left(x_{i}, x_{j}\right)\right] \alpha_{i} \alpha_{j}
$$

is positive definite on $\boldsymbol{R}^{n}$.

Familiar examples are Brownian motions with parameter space $\boldsymbol{S}^{n}$ and with parameter space $\boldsymbol{R}^{n}$.

II. Let $\boldsymbol{P}^{n}$ be the $n$-dimensional real projective space, and let $x=\left(x_{1}, x_{2}, \cdots, x_{n}, x_{0}\right)$ be a point in $\boldsymbol{P}^{n}$ expressed in terms of the homogeneous coordinate. We introduce a local coordinate $\pi$ of $\boldsymbol{P}^{n}$, which is defined as follow:

$\pi$ is a coordinate of $\boldsymbol{P}^{n}-\left\{x ; x_{0}=0\right\}$ such that 


$$
\pi(x)=\left(\frac{x_{1}}{x_{0}}, \cdots, \frac{x_{n}}{x_{0}}\right), \quad x \in \boldsymbol{P}^{n}-\left\{x ; x_{0}=0\right\} .
$$

Set $\tilde{x}=\pi^{-1}(x), x \in \boldsymbol{R}^{n}$, and let $*$ denote the inversion mapping of $P^{n}$, that is, * maps $\boldsymbol{P}^{n}$ onto

$\mathfrak{N}=\left\{(n-1)\right.$-dimensional hyperplane in $\left.\boldsymbol{P}^{n}\right\}$ in such a way that

$$
x \mapsto x^{*}=\left\{y \in \boldsymbol{P}^{n} ; x \cdot{ }^{t} y=x_{1} y_{1}+\cdots+x_{n} y_{n}+x_{0} y_{0}=0\right\}, \quad x \in \boldsymbol{P}^{n} .
$$

For convenience we introduce another coordinate system of $\boldsymbol{P}^{n}$. Let $\boldsymbol{S}^{n}$ be viewed as a two folded covering space of $\boldsymbol{P}^{n}$. For an element $x$ of $\boldsymbol{P}^{n}$, we define the polar coordinate $(q ; \phi)$ of $x$ in the following manner:

$q \in \boldsymbol{S}^{n-1}$ is the direction of the vector $\left(x_{1}, \cdots, x_{n}\right)$

and

$$
\cos \phi=x_{0} /\left(x_{1}^{2}+\cdots+x_{0}^{2}\right)^{1 / 2}, \quad 0 \leq \phi \leq \pi .
$$

With this notation $\pi(x)$ and $\pi\left(x^{*}\right)$ may be expressed in the form

$$
\pi(x)=(q, \tan \phi),
$$

and

(33) $\pi\left(x^{*}\right)=\left\{\begin{array}{ll}\text { the hyperplane which contains a point } & (-q, \cot \phi) \\ \text { and is perpendicular to the vector } & (-q, \cot \phi)\end{array}\right\}$

III. We start with a simple construction of the $\boldsymbol{S}^{n}$-parameter Brownian motion. Let $O$ be the origin of $\boldsymbol{S}^{n}$ and let $d_{s}$ be the geodesic metric. Associate with a point $A$ of $S^{n}$ is a semi-sphere $S(A)$ defined by

$$
S(A)=\left\{B \in S^{n} ; d_{s}(A, B) \leq \frac{\pi}{2}\right\}
$$

Let $d S$ denote the uniform measure on $S^{n}$, and let $\{\xi \sqrt{d S}\}$ be a white noise on $\left(\boldsymbol{S}^{n}, d S\right)$. We define a random variable $\tilde{B}_{s}(A)$ for any point $A$ of $S^{n}$ as follows:

$$
\tilde{B}_{s}(A)=\sqrt{\frac{\pi}{\sigma_{n}}} \int_{S(A)} \xi_{M} \sqrt{d S(M)},
$$

where $\sigma_{n}=\int_{S_{n}} d S$, and define a new random variable 


$$
B_{s}(A)=\tilde{B}_{s}(A)-\tilde{B}_{s}(O) \text {. }
$$

Then we get easily the following theorem.

ThEOREM 5. The Gaussian system $\left\{B_{s}(A) ; A \in S^{n}\right\}$ is the Brownian motion with parameter space $\left(S^{n}, d_{s}\right)$.

The domain of the integral (35) never contains any set of antipodal pairs of positive measure. So we may take a new random measure $\left\{\xi^{\prime} \sqrt{d S}\right\}$ on $\boldsymbol{P}^{n}=\boldsymbol{S}^{n} / \sim$ (where $A \sim B$ means that $A$ and $B$ is an antipodal pair) as

$$
\xi_{M}^{\prime}=\frac{1}{\sqrt{2}}\left(\xi_{M}-\xi_{\tilde{M}}\right)
$$

where $M \sim \tilde{M}$.

Then the integral (35) is expressible as

$$
\begin{aligned}
\tilde{B}_{s}(A) & =\sqrt{\frac{\pi}{\sigma_{n}}} \int_{P^{n}\left(A^{*}\right)} \xi_{M}^{\prime} \sqrt{d S(M)} \\
& =\sqrt{\frac{\pi}{2 \sigma_{n}}}\left(\int_{S(A)} \xi_{M} \sqrt{d S(M)}-\int_{S(A) c} \xi_{M} \sqrt{d S(M)}\right) .
\end{aligned}
$$

The meaning of the first integral of $\left(35^{\prime}\right)$ is that the domain of integration is $\boldsymbol{P}^{n}-A^{*}$ and the orientation of $\boldsymbol{P}^{n}-A^{*}$ is given by continuation from that of the origin $O$ of $\boldsymbol{S}^{n}$.

We can easily get the following symmetry of the Brownian motion $\left\{\tilde{B}_{s}(A) ; A \in \boldsymbol{S}^{n}\right\}$ that can be described by the rotation group.

THEOREM 6. It holds that

$$
\tilde{B}_{s}(A g, \omega)=\tilde{B}_{s}(A, g \circ \omega),
$$

for any element $g$ of the rotation group $S O(n+1, R)$.

Observe the expression (35') restricting $A$ to the space $\boldsymbol{P}^{n}-O^{*}$, and apply the local coordinate map. Then we can rewrite the formula (35') in the form

$$
\tilde{B}_{s}(\tilde{a})=\sqrt{\frac{\pi}{\sigma_{n}}} \int_{P^{n}\left(\tilde{a}^{*}\right)} \xi_{M}^{\prime} \sqrt{d S(M)}, \quad a \in \boldsymbol{R}^{n}
$$

The process $\left\{\tilde{B}_{s}(\tilde{a}) ; a \in \boldsymbol{R}^{n}\right\}$ defined by (39) is the Brownian motion with parameter space $\left(\boldsymbol{R}^{n}, d_{\pi}=\pi_{*}\left(d_{s}\right)\right)$. We want to form a Brownian 
motion with parameter space $\left(\boldsymbol{R}^{n},\|\cdot\|\right)$ from this process $B_{s}(\cdot)$ by a change of the associated measure. We note that the Euclidean metric $\|\cdot\|$ is invariant under the action of the Euclidean motion group $M(n)$, while $d_{\pi}$ is invariant under the action of $S O(n+1)$. We, then, observe that a matrix representation of $M(n)$ as follows:

$$
\begin{aligned}
M(n)= & \left\{g ; g=\left(\frac{h}{a} \mid \frac{\widetilde{0}}{1}\right) \in S L(n+1, \boldsymbol{R}),\right. \\
& \left.h \in S O(n), a \in \boldsymbol{R}^{n}, \widetilde{0}={ }^{t}(0, \cdots, 0)\right\} .
\end{aligned}
$$

where the action by $g=\left(\frac{h}{a} \mid \frac{\tilde{0}}{1}\right)$ on $x \in \boldsymbol{R}^{n}$ is such that

$$
x \cdot g=x \cdot h+a .
$$

$S O(n+1)$ and $M(n)$ have $S O(n)$ as a common subgroup under which both $d_{\pi}$ and $\|\cdot\|$ are invariant. We further notice that the relation (31), $a \leftrightarrow a^{*}$, links the parameter space of a Brownian motion and the parameter space of a white noise. Thus, so far as a Brownian motion defined by the analogous formula $\left(35^{\prime}\right)$ is invariant under the action of $M(n)$, the measure $d \zeta$ by which the Brownian motion with parameter space $\left(\boldsymbol{R}^{n},\|\cdot\|\right)$ is defined, has to be invariant under the action of $M^{*}(n)$. In our case

$$
M^{*}(n)=\left\{{ }^{t} g ; g \in M(n)\right\} .
$$

By a short calculus the measure $d \Psi$ on $P^{n}$ must become the following form on $\boldsymbol{R}^{n}$ :

$$
\pi_{*}(d \Psi)=\frac{C}{r^{n+1}} d x
$$

where $C$ is a normalizing constant which is to be given in the proof of the next theorem. For notational simplicity, we write $\pi_{*}(d \Psi)$ simply as $d \Psi$ again.

Set,

$$
B(a)=\mathrm{p} . \mathrm{v} \cdot\left(\int_{P^{n}\left(\tilde{a}^{*}\right)}-\int_{P^{n}\left(\tilde{0}^{*}\right)}\right) \xi_{M} \sqrt{d \mathrm{U}(M)} .
$$

Then holds the next theorem.

Theorem 7. A Process $\left\{B(a) ; a \in \boldsymbol{R}^{n}\right\}$ given by the form (42) is the 
$\boldsymbol{R}^{n}$-parameter Brownian motion in the sense of Lévy, that is, $\left\{B(a) ; a \in \boldsymbol{R}^{n}\right\}$ hold the conditions (26) and (27).

Proof. The domain of the integral (42) does not contain a non-trivial disc $D_{\varepsilon}$ with center 0 and radius $\varepsilon$. Then

$$
E|B(a)|^{2} \leq \int \chi_{\left(D_{\varepsilon}\right)} c d=C \int_{\varepsilon}^{\infty} \frac{d r}{r^{2}}=C(1 / \varepsilon) .
$$

The integral (42) is therefore well defined.

By the construction of $\{\xi \sqrt{d \bar{\varphi}}\}$ the variance $\mathrm{E}|B(a)-B(b)|^{2}$ of the difference is a function only of the distance between $a$ and $b$. It is now enough for us to prove that the variance $\mathrm{E}|B(a)-B(b)|^{2}$ is proportional to the distance $\|a-b\|$.

Take such $a$ and $b$ as

$$
\begin{aligned}
& a=\left(r, r \tan \psi_{a}, 0, \cdots, 0\right), \\
& b=\left(r, r \tan \psi_{b}, 0, \cdots, 0\right) .
\end{aligned}
$$

Then $a=r / \cos \psi_{a}, b=r / \cos \psi_{b}$ and

$$
a^{*} \cap b^{*}=\left\{x=\left(x_{1}, \cdots, x_{n}\right) ; x_{1}=r_{0}=1 / r, x_{2}=0\right\} .
$$

The solid angle of the domain limited by two coaxial cones of vertical angle $\theta$ and $\theta+d \theta$ is proportional to $d \theta$ and is as large as $2 Q_{\theta} d \theta$. We therefore have

$$
\begin{aligned}
E|B(a)-B(b)|^{2} & =\frac{C}{\sigma_{n}}\left\{\int_{-\pi / 2}^{0}-\int_{0}^{\pi / 2}\right\} Q_{\theta} d \theta \int_{r_{0} \cos \psi_{a} / \cos \left(\theta-\psi_{a}\right)}^{r_{0} \cos \psi_{b} / \cos \left(\theta-\psi_{b}\right)} \frac{d r}{r^{2}} \\
& =\frac{C}{r_{0} \sigma_{n-1}} \int_{-\pi / 2}^{\pi / 2} Q_{\theta}\left|\frac{\cos \left(\theta-\psi_{b}\right)}{\cos \psi_{b}}-\frac{\cos \left(\theta-\psi_{a}\right)}{\cos \psi_{a}}\right| d \theta \\
& =\frac{1}{r_{0}}\left|\tan \psi_{a}-\tan \psi_{b}\right| \times \frac{C}{\sigma_{n-1}} \int_{-\pi / 2}^{\pi / 2} Q_{\theta}|\sin \theta| d \theta=\|a-b\|,
\end{aligned}
$$

where $C$ is the normalizing constant such that

$$
\frac{C}{\sigma_{n-1}} \int_{-\pi / 2}^{\pi / 2} Q_{\theta}|\sin \theta| d \theta=1
$$

We are now ready to show the symmetry of the above constructed Brownian motion that comes from the structure of $\Omega$-space.

THEOREM 8. For any element $g$ of $M(n)$, we have 


$$
B(a g, \omega)=B\left(a, \hat{g}_{0} \omega\right)-B\left(O, \hat{g}_{0} \omega\right)
$$

where $\hat{g}$ is the transposed inverse matrix of $g$ in the matrix representation (40) of $M(n)$.

Let $V(a)$ be the domain of the integral (41). Then we can see that

$$
\pi\left(\widetilde{V(a)^{*}}\right)=\left\{\begin{array}{l}
\text { the collection of hyperplanes of } \boldsymbol{R}^{n} \text { which } \\
\text { separate two point } O \text { and } a
\end{array}\right\} .
$$

That is, our white noise representation of the $\boldsymbol{R}^{n}$-parameter Brownian motion is equivalent to that of Ченцов ([16]).

\section{§5. Radon transform and Wiener integral}

In the following, the dimension $n$ is always assumed to be odd.

DEFINITION 3. Let $\mathscr{S}\left(\boldsymbol{R}^{n}\right)$ be the Schwartz space of rapidly decreasing real $C^{\infty}$-functions. The Radon transform $\check{f}$ of $f$ in $\mathscr{S}\left(\boldsymbol{R}^{n}\right)$ is the function on $\boldsymbol{R}^{n} \times \boldsymbol{R}$ defined by:

$$
\check{f}(\xi, p)=\frac{1}{\sqrt{2 \pi}} \int_{-\infty}^{\infty} \tilde{f}(\alpha \xi) e^{-i \alpha p} d \alpha, \quad \xi \in \boldsymbol{R}^{n}, p \in \boldsymbol{R},
$$

where $\tilde{f}$ is the Fourier transform of $f$ :

$$
\tilde{f}(\xi)=\left(\frac{1}{2 \pi}\right)^{n / 2} \int_{\boldsymbol{R}^{n}} f(x) e^{i\langle\xi, x\rangle} d x,
$$

the bracket $\langle\cdot, \cdot\rangle$ being the inner product in $\boldsymbol{R}^{n}$.

For notational convenience, we write the Radon transform simply as

$$
\check{f}(\xi, p)=\int_{\langle\xi, x\rangle=p} f(x) d x .
$$

Here is an important remark that should be emphasized. One can see an interesting and beautiful similarity, in expression by integral, between the Radon transform of a function on $\boldsymbol{R}^{n}$ and the white noise representation of $\boldsymbol{R}^{n}$-parameter Brownian motion through the relation

$$
\pi\left(\tilde{\xi}^{*}\right)=\{x ;\langle x, \xi\rangle=-1\} .
$$

Тнеоrem 9. (И. М. Гельфанд, М. И. Граев и Н.Я. Виленкин [2b]). In case the dimension $n$ is odd, setting $m=(n-1) / 2$, we have 


$$
\int_{R^{n}} f(x) h(x) d x=\frac{1}{2 \sigma_{n-1}} \int_{S^{n-1}} \int_{-\infty}^{\infty} \check{f_{p}^{(m)}}(q, p) \check{h}_{p}^{(m)}(q, p) d q d p
$$

$f, h \in \mathscr{S}\left(\boldsymbol{R}^{n}\right)$, where $\check{f}_{p}^{(m)}=\left(\frac{\partial}{\partial p}\right)^{m} \breve{f}, q \in \boldsymbol{S}^{n-1}$ and $d q$ is the uniform measure on $\boldsymbol{S}^{n-1}$.

Proof. Notice the fact that the function $\check{f}(\xi, p)$ is real.

$$
\begin{aligned}
\int_{S^{n-1}} \int_{-\infty}^{\infty} & \check{f}_{p}^{(m)}(q, p) \check{h}_{p}^{(m)}(q, p) d q d p \\
& =\frac{1}{2 \pi} \int_{S^{n-1}} \int_{-\infty}^{\infty}\left[\int_{-\infty}^{\infty}(-i \alpha)^{m} \tilde{f}(\alpha q) e^{-i \alpha p} d \alpha\right]\left[\int_{-\infty}^{\infty}(i \beta)^{m} \overline{\tilde{h}(\beta q)} e^{i \beta p} d \beta\right] d q d p \\
& =\int_{S^{n-1}} \int_{-\infty}^{\infty} \int_{-\infty}^{\infty}(\alpha \beta)^{m} \tilde{f}(\alpha q) \overline{\tilde{h}(\beta q)} \delta(\alpha-\beta) d \alpha d p d q \\
& =\int_{S^{n-1}} \int_{-\infty}^{\infty} \alpha^{n-1} \tilde{f}(\alpha q) \overline{\tilde{h}(\alpha q)} d \alpha d q=2 \sigma_{n-1} \int_{\boldsymbol{R}^{n}} \tilde{f}(\xi) \overline{\tilde{h}(\xi)} d \xi \\
& =2 \sigma_{n-1} \int_{R^{n}} f(x) h(x) d x .
\end{aligned}
$$

Set

$$
(R f)(\eta)=\check{f_{p}^{(m)}}\left(\frac{\eta}{\|\eta\|}, \frac{-1}{\|\eta\|}\right), \quad \text { for } \boldsymbol{R}^{n}-\{0\}
$$

Then the equation (47) is expressible as

$$
\int_{R^{n}} f(x) h(x) d x=\int_{P^{n}\left(0^{*}\right)}(R f)(R h) d \Psi^{\prime}, \quad \text { where } \quad d \mathrm{\Psi}^{\prime}=\frac{d r d q}{r^{2} \sigma_{n-1}} \propto d \mathrm{\Psi} .
$$

THEOREM 10. The map $R$ can be extended to an isometry between $L^{2}\left(\boldsymbol{R}^{n}, d x\right)$ and $L^{2}\left(\boldsymbol{R}^{n}, d \mathbf{\Psi}^{\prime}\right)$.

Proof. By the equation $\left(49^{\prime}\right)$ the map $R$ is an into isometry. Let $D^{\vee}$ be a space of all $C^{\infty}\left(\boldsymbol{R}^{n}\right)$-functions $F$ with compact support $K_{F}$ and let $Q$ be an operator on $D^{\vee}$ defined by

$$
\begin{aligned}
F(\xi) \mapsto(Q F)(y)= & \frac{1}{2 \sigma_{n-1}}\left(\frac{1}{2 \pi}\right)^{(n+1) / 2} \int_{S^{n-1}} \int_{-\infty}^{\infty} \int_{\beta \in R}(i \beta)^{m} \\
& \times F(q, r) \exp \left(-\frac{i \beta}{r}-i \beta\langle y, q\rangle\right) \frac{d r}{r^{2}} d \beta d q,
\end{aligned}
$$

where $(q, r)$ is the polar coordinate of $\xi$. The operator $Q$ takes values in $L^{2}\left(\boldsymbol{R}^{n}, d x\right)$. If $F, G$ are in $D^{\vee}$, then 


$$
\begin{aligned}
& \int_{R^{n}}(Q F)(y)(Q G)(y) d y \\
&= \frac{1}{2 \sigma_{n-1}}\left(\frac{1}{2 \pi}\right)^{n+1} \int \cdots \int\left[(i \beta)\left(-i \beta^{\prime}\right)\right]^{m} F(q, r) G\left(q^{\prime}, r^{\prime}\right) \\
& \times \exp \left(-\frac{i \beta}{r}+\frac{i \beta^{\prime}}{r^{\prime}}-i\left\langle y, \beta q-\beta^{\prime} q^{\prime}\right\rangle\right) d y \frac{d r}{r^{2}} \frac{d r^{\prime}}{r^{\prime 2}} \frac{d(\beta q)}{\beta^{n-1}} d \beta^{\prime} d q^{\prime} \\
&= \frac{1}{4 \pi \sigma_{n-1}} \int \cdots \int F\left(q^{\prime}, r\right) G\left(q^{\prime}, r^{\prime}\right) \exp \left(-i \beta^{\prime}\left(\frac{1}{r}-\frac{1}{r^{\prime}}\right)\right) \frac{d r}{r^{2}} \frac{d r^{\prime}}{r^{\prime 2}} d \beta^{\prime} d q \\
&= \frac{1}{2 \sigma_{n-1}} \iint F(q, r) G(q, r) \frac{d r}{r^{2}} d q=\int F G d \mathrm{Y}^{\prime} .
\end{aligned}
$$

This shows that $Q$ can be extended to an isometry from $L^{2}\left(\boldsymbol{R}^{n}, d \mathrm{U}^{\prime}\right)$ into $L^{2}\left(\boldsymbol{R}^{n}, d x\right)$. For $f \in L^{2}\left(\boldsymbol{R}^{n}, d x\right)$

$$
\begin{aligned}
(Q R f)(y)= & \frac{1}{2 \sigma_{n-1}}\left(\frac{1}{2 \pi}\right)^{(m+1)} \int \cdots \int \exp \left(-\frac{i \beta}{r}-i \beta\langle y, q\rangle\right)(i \beta)^{m}(-i \alpha)^{m} \\
& \times \tilde{f}(\alpha q) \exp \left(-\frac{\alpha}{r}\right) d \beta d q d \alpha \frac{d r}{r^{2}} \\
= & \frac{1}{2 \sigma_{n-1}}\left(\frac{1}{2 \pi}\right)^{n / 2} \iint \tilde{f}(\alpha q) \alpha^{(n-1)} e^{-i\langle y, \alpha q\rangle} d \alpha d q \\
= & \left(\frac{1}{2 \pi}\right)^{n / 2} \int_{R^{n}} \tilde{f}(x) e^{-i\langle y, x\rangle} d x=f(y) .
\end{aligned}
$$

That is, $Q R$ is the identity map of $L^{2}\left(\boldsymbol{R}^{n}, d x\right)$. Hence the operator $R$ is an onto map.

q.e.d.

We use the same symbol to express this extension of $R$. The next proposition can be easily be proved.

Proposition 2. For any nonzero real number $\alpha$, we have

$$
\check{f}(\alpha \xi, \alpha p)=(1 / \alpha) \check{f}(\xi, p),
$$

and

$$
\check{f}_{p}^{(m)}(\alpha \xi, \alpha p)=(1 / \alpha)^{(m+1)} \check{f}(\xi, p) .
$$

DEFINITION 4. For any element $f$ of $L^{2}\left(\boldsymbol{R}^{n}, d x\right)$, the Wiener integral of $f$ is defined by:

$$
W(f, a)=\int_{P^{n}\left(a^{*}\right)}(R f)(\eta) \xi_{\eta} \sqrt{d \mathrm{U}^{\prime}(\eta)}, \quad a \in \boldsymbol{R}^{n} .
$$

Proposition 3. For any $f$ and $h$ of $L^{2}\left(\boldsymbol{R}^{n}, d x\right)$, we have 


$$
\mathrm{E}(W(f, a) W(h, a))=\int_{R^{n}} f(x) h(x) d x .
$$

The special linear group $S L(n+1, R)$ appears as the structure group of the quasi-white noise by which we define the Brownian motion. We will study this group as the symmetry group of the Wiener integral (52) in the following two section.

\section{§6. Infinite dimensional rotation group $0^{\infty}$ and its finite dimensional subgroup}

Let $\mathscr{H}$ be a real Hilbert space and $\mathscr{D}$ be a dense nuclear subspace of $\mathscr{H}$. Consider the collection $O(\mathscr{D})$ given by

$$
O(\mathscr{D})=\{T ; \text { orthogonal linear operator on } \mathscr{H}, T \mathscr{D} \subset \mathscr{D}\} \text {. }
$$

It forms a group under the usual product, indeed it is a transformation group action on $\mathscr{D}$.

DEFINITION 5. The group $O(\mathscr{D})$ is called the infinite dimensional rotation group.

We sometime write it as $O^{\infty}$ instead of $O(\mathscr{D})$. This group $O^{\infty}$ plays a very important role when we discuss the so-called Fock decomposition of the Hilbert space $L^{2}\left(\mathscr{D}^{*}, P\right)$ :

$$
L^{2}\left(\mathscr{D}^{*}, P\right)=\operatorname{Exp} \mathscr{H},
$$

where $\mathscr{D}^{*}$ is the dual space of $\mathscr{D}$ (c.f. T. Hida [5c] and Y. Umemura [13]). The projective limit of the finite dimensional rotation group $S O(n)$

$$
O_{\infty}=\lim _{n} S O(n),
$$

is merely a small subgroup of $O^{\infty}$. We are not concerned with this group $O^{\infty}$ itself, but we investigate an important subgroup of $O^{\infty}$ which is not contained in $O_{\infty}$.

\section{The case of ordinary Brownian motion}

In this subsection we summarize what is known concerning the relationship between a certain subgroup of $O^{\infty}$ and the projective invariance of the ordinary Brownian motion.

A finite dimensional subgroup $F_{1}$ of $O^{\infty}$ comes from the Brownian motion in the following manner. Set, 


$$
\mathscr{D}_{1}=\left\{f ; f \in C^{\infty}\left(\boldsymbol{R}^{1}\right),(1 / x) f(1 / x) \in C^{\infty}\left(\boldsymbol{R}^{1}\right)\right\} \text {. }
$$

Consider the following two one-paramer subgroups of $O\left(\mathscr{D}_{1} \cap L^{2}\left(\boldsymbol{R}^{1}, d x\right)\right)$ :

(S) $S(t)$ : Shift,

$$
(S(t) f)(x)=f(x+t), \quad t \in R,
$$

(D) $D(t):$ Dilation (or Tension),

$$
(D(t) f)(x)=e^{t / 2} f\left(e^{t} x\right), \quad t \in \boldsymbol{R} .
$$

The infinitesimal operators correspond to the one-parameter groups $(S)$ and $(D)$ are $(s)$ and $(d)$ respectively:

$$
\begin{gathered}
s=\frac{d}{d x}, \\
\tau=\frac{1}{2} I+x \frac{d}{d x},
\end{gathered}
$$

where $I$ is the identity operator on $L^{2}\left(\boldsymbol{R}^{1}, d x\right)$. The Lie product $[s, \tau]$ of $s$ and $\tau$ is equal to $s$, so that $\{s, \tau\}$ forms a base of a Lie algebra generated by them.

Consider the map $J$ given by

$$
(J f)(x)=\frac{1}{x} f\left(-\frac{1}{x}\right) \quad \text { for } f \in D_{1},
$$

and introduce the adjoint operators of $s$ and $\tau$ with respect to $J$ :

$$
\operatorname{Ad}(J) s=\left(J^{-1} s J\right)=x I+x^{2} \frac{d}{d x}
$$

and

$$
\operatorname{Ad}(J) \tau=-\tau
$$

Then we are given a new operator

$$
\kappa=x I+x^{2} \frac{d}{d x} .
$$

Since $[s, \kappa]=2 \tau$ and $[\tau, \kappa]=\kappa$ hold, $\{s, \tau, \kappa\}$ is a base of a Lie algebra of differential operators on $D_{1}$. Corresponding to $\kappa$ we consider a new oneparameter subgroup $K(t)$ of $O^{\infty}$ such that 


$$
(K(t) f)(x)=\left(\left(J^{-1} S(t) J\right) f\right)(x)=\frac{1}{1-t x} f\left(\frac{1}{1-t x}\right), \quad t \in \boldsymbol{R} .
$$

Then the operator $\kappa$ is the infinitesimal generator of the operator $(K)$.

The one-parameter subgroup $(S),(D)$ and $(K)$ give an unitary representation $T_{g}$ of $g S L(2, R)$ such that:

$$
T_{g} f(x)=\frac{1}{\beta x+\delta} f\left(\frac{\alpha x+\gamma}{\beta x+\delta}\right)
$$

where $g=\left(\begin{array}{l}\alpha, \beta \\ \gamma, \delta\end{array}\right) \in S L(2, R)$ and $f \in L^{2}(\boldsymbol{R}, d x)$.

With the operators $(S),(D)$ and $(K)$, we can associate one-parameter subgroups of $S L(2, R)$ in such a manner that:

$$
S(t) \simeq\left(\begin{array}{l}
1,0 \\
t, 1
\end{array}\right), \quad D(t) \simeq\left(\begin{array}{cc}
e^{t / 2}, & 0 \\
0, e^{-t / 2}
\end{array}\right), \quad K(t) \simeq\left(\begin{array}{rr}
1,-t \\
0, & 1
\end{array}\right)
$$

In addition we have

$$
J \simeq\left(\begin{array}{r}
0,1 \\
-1,0
\end{array}\right)
$$

By the Bochner-Minlos theorem (c.f. [2a]) one can introduce a paobability measure $P$ on $\mathscr{D}_{1}{ }^{*}$, with respect to this probability measure

$$
B(t, \omega)=\left\langle\chi_{[0, t)}, \omega\right\rangle, \quad \omega \in \mathscr{D}_{1}^{*},
$$

is a version of the ordinary Brownian motion. Then each one of the one-parameter group $(S),(D)$ and $(K)$ defines a flow on $\left(\mathscr{D}_{1}^{*}, P\right)$. These three flows, acting on $\Omega$-space, can be switched to the transformations acting on the sample paths through the above expression (62). With this, the projective invariance, due to P. Lévy, of the Brownian motion can easily be interpreted (c.f. T. Hida, I. Kubo, H. Nomoto and H. Yoshizawa [6]).

II. Let $\mathscr{D} \subset L^{2}\left(\boldsymbol{R}^{n}, d x\right) \subset \mathscr{D}^{*}$ be any Gelfand triple. By the definition of the Wiener integral (52) we get a probability space $\left(\mathscr{D}^{*}, P\right.$ ) which is taken to be an $\Omega$-space for the Brownian motion with parameter space $\left(\boldsymbol{R}^{n},\|\cdot\|\right)$.

We wish to extend the results in I to the multi-parameter cases. As soon as we come to the higher dimensional space, say $\boldsymbol{R}^{n}$, one meets very much complicated structure and finds that $\boldsymbol{R}^{n}$ would be better to 
be viewed as $\boldsymbol{P}^{n}$ by the use of the local coordinate $\pi$. Under such a view point the relationship between the parameter space structure and the projective invariance of the Brownian motion can be well be recognized.

$1^{\circ}$. The first problem to be discussed here is how to generalize the operator $J$ to the multi-parameter case. If directions in $\boldsymbol{R}^{n}$ are taken into account, one is naturally led to such operators $J_{i}$ 's that

(J) $J_{i} f\left(x_{1}, \cdots, x_{n}\right)=\left(\frac{1}{x_{i}}\right)^{(n+1) / 2} f\left(\frac{x_{1}}{x_{i}}, \cdots, \frac{x_{i-1}}{x_{i}}, \frac{1}{x_{i}}, \frac{x_{i+1}}{x_{i}}, \cdots, \frac{x_{n}}{x_{i}}\right)$.

The nuclear space on which the $J_{i}$ act must be

$$
\mathscr{D}_{n}=\left\{f ; f \in C^{\infty}\left(\boldsymbol{R}^{n}\right), J_{i} f \in C^{\infty}\left(\boldsymbol{R}^{n}\right) \text { for } i=1, \cdots, n\right\} \text {. }
$$

This space $\mathscr{D}_{n}$ can be identified with the space $C^{\infty}\left(\boldsymbol{P}^{n}\right)$ in the usual manner. Now consider the following operators defined on $\mathscr{D}_{n}$ :

$$
\begin{gathered}
s_{i}=\frac{\partial}{\partial x_{i}}, \quad i=1, \cdots, n, \\
\tau_{i}=\frac{1}{2} I+x_{i} \frac{\partial}{\partial x_{i}}, \quad i=1, \cdots, n .
\end{gathered}
$$

Then their Lie products are the following:

$$
\begin{aligned}
{\left[s_{i}, \tau_{j}\right] } & =0, & & \text { if } i \neq j . \\
& =s_{i}, & & \text { if } i=j .
\end{aligned}
$$

The adjoint operators of the $s_{j}$ with respect to $J_{i}$ are introduced and are of the form

$$
\begin{aligned}
\operatorname{Ad}\left(J_{i}\right) s_{j} & =x_{i} \frac{\partial}{\partial x_{i}}, \quad \text { if } i \neq j \\
& =\frac{n+1}{2} x_{i} I+x_{i} \sum_{k=1}^{n} x_{k} \frac{\partial}{\partial x_{k}}, \quad \text { if } i=\jmath .
\end{aligned}
$$

Define new operators

$$
\begin{gathered}
\kappa_{i}=\operatorname{Ad}\left(J_{i}\right) s_{i} . \\
s_{i j}=\operatorname{Ad}\left(J_{i}\right) s_{j}, \quad \text { for } i \neq j .
\end{gathered}
$$

The commutation relations of the operators $(s),(d),(k)$ and $\left(s^{\prime}\right)$ immediately prove the following.

Proposition 4. The operators $\left\{s_{i}, \tau_{i}, \kappa_{i}, s_{i j}\right\}$ generate a Lie algebra $\mathfrak{f}_{n}$ isomorphic to $\mathfrak{g l}(n+1, R)$. 
$2^{\circ}$. Now we give the explicite form of one-parameter subgroups $(S),(D),(K)$ and $\left(S^{\prime}\right)$ with infinitesimal generators $(s),(d),(k)$ and $\left(s^{\prime}\right)$ respectively, and express them by $(n+1)$-dimensional matrices as follows :

(S) $S_{i}(t):$ Shift

$$
\left(S_{i}(t) f\right)(x)=f\left(x_{1}, \cdots, x_{i-1}, x_{i}+t, x_{i+1}, \cdots, x_{n}\right), \quad t \in \boldsymbol{R}, f \in L^{2}\left(\boldsymbol{R}^{n}, d x\right),
$$

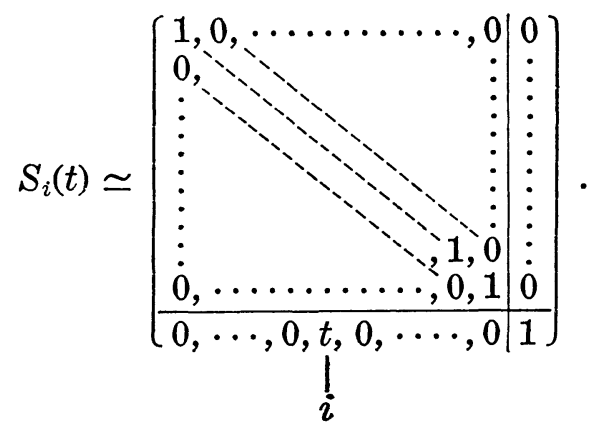

(D) $\quad D_{i}(t):$ Dilation

$$
\begin{aligned}
& \left(D_{i}(t) f\right)(x)=e^{t / 2} f\left(x_{1}, \cdots, x_{i-1}, e^{t} x_{i}, x_{i+1}, \cdots, x_{n}\right),
\end{aligned}
$$

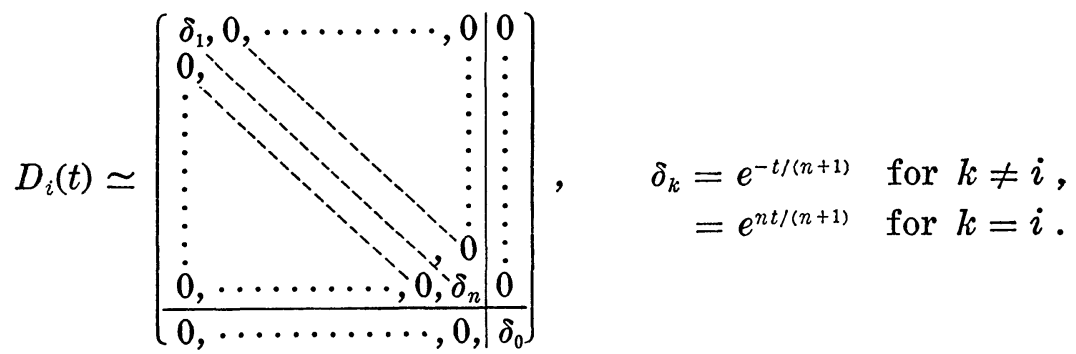

(K) $\quad\left(K_{i}(t) f\right)(x)$

$$
\begin{aligned}
& =\frac{1}{1-t x_{i}} f\left(\frac{x_{1}}{1-t x_{i}}, \cdots, \frac{x_{i-1}}{1-t x_{i}}, \frac{-1}{1-t x_{i}}, \frac{x_{i+1}}{1-t x_{i}}, \cdots, \frac{x_{n}}{1-t x_{i}^{i}}\right),
\end{aligned}
$$

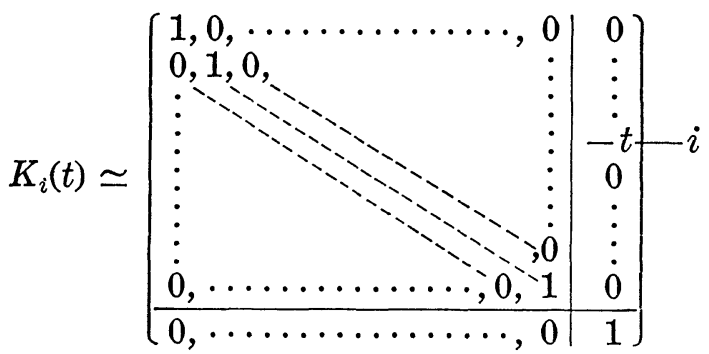




$$
\left(S_{i j}(t) f\right)(x)=f\left(x_{1}, \cdots, x_{i-1}, x_{i}+t x_{j}, x_{i+1}, \cdots, x_{n}\right), \quad i \neq j,
$$

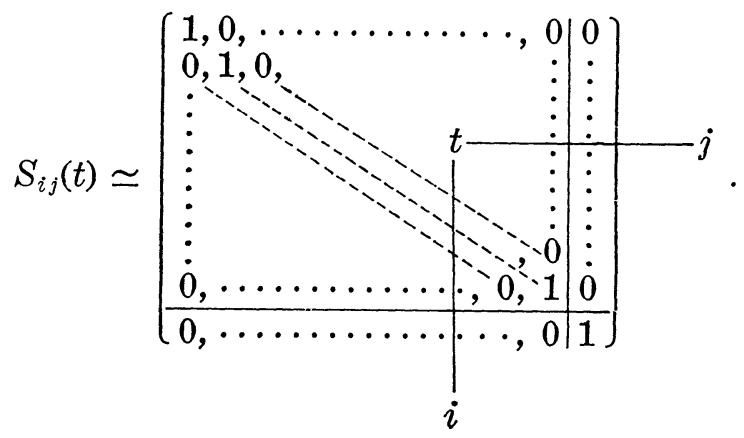

And the matrix representation of the inversion operators $(J)$ are

$(J)$

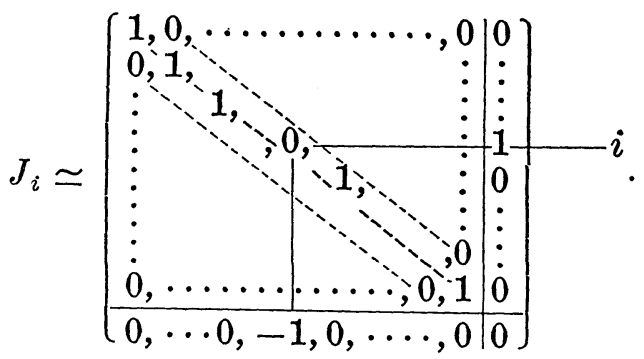

Obviously $(S),(D),(K)$ and $\left(S^{\prime}\right)$ are elements of $S L(n+1, R)$ and generate the entire group $S L(n+1, \boldsymbol{R})$. The space $D_{n} \cap L^{2}\left(\boldsymbol{R}^{n}, d x\right)$ is nuclear and is invariant under these unitary operators. Then it holds that

$$
O^{\infty} \supset F_{n} \simeq S L(n+1, R),
$$

where $F_{n}$ is the operator group which is generated by the operator $(S)$, $(D),(K)$ and $\left(S^{\prime}\right)$.

Let $T_{g}$ be the unitary representation of $S L(n+1, R)$ defined by $F_{n}$.

$3^{\circ}$. We have so far obtained a quasi-regular (continuous) unitary representation $T_{g}$ of the group $S L(n+1, R)$ on $L^{2}\left(\boldsymbol{R}^{n}, d x\right)$. One may now ask probabilistic meanings of this representation $T_{g}$. As we have observed in $\S 4$, the white noise $\left\{\xi_{x} \sqrt{d \Psi(x)}\right\}$ on $\boldsymbol{P}^{n}$ is invariant under the action of the Euclidean motion group $M(n)$. The group $M(n)$ is only a subgroup of $S L(n+1, R)$ (see (40)) under which the relation between $x$ and $x^{*}$ (see (31)) in $\boldsymbol{P}^{n}$ is kept invariant. Accordingly it is natural to consider the system $\left\{\xi_{x} \sqrt{d \bar{U}}\right\}$ as an $S L(n+1, R)$-quasi-white noise on $\boldsymbol{P}^{n}$. We, therefore, consider the unitary representation $U_{g}$ of $S L(n+1, \boldsymbol{R})$ on $L^{2}\left(\boldsymbol{P}^{n}, d \Psi\right)$ by using the procedure in $\S 3$. On the other hand, we 
have considered in $\S 5$ that the modified Radon transform $R$ gives us the relation between the quasi-white noise $\left\{\xi_{x} \sqrt{d \bar{U}}\right\}$ and the Brownian motion $\{B(a)\}$. Combining these facts we get our main result asserting that this operator $R$ links these two representations $T_{g}$ and $U_{g}$ in the following manner.

MAIN THEOREM. In the case of odd dimension

$$
R T_{g}=U_{\hat{g}} R, \quad \text { for any } g \text { in } S L(n+1, R),
$$

where $\hat{g}$ is ${ }^{t} g^{-}$in the matrix form (40).

That is, the modified Radon transform $R$ is nothing but the intertwining operator between two representations $T_{g}$ and $U_{t_{g-1}}$ of $S L(n+1, R)$. We shall prove the main theorem in the next section.

In the one-dimensional case, the Radon transform is rather trivial, namely

$$
(R f)(x)=f\left(-\frac{1}{x}\right)
$$

If we consider it together with the density $d \mathrm{Y}^{\prime} / d x=1 /\left(x^{2}\right), R$ becomes equal to $J$. Therefore it is difficult to grasp the beautiful relation (66) in the case of the ordinary Brownian motion. Our main theorem ties up three fact, the representation of the Brownian motion by the integral with respect to the white noise, the Wiener integral and the subgroup $F_{n}$ of $O^{\infty}$. So it should be, the author hopes, a key point of the theory of multi-parameter white noise analysis.

\section{$\S 7$. Proof of the main theorem}

Since the full group $S L(n+1, R)$ is generated by $(S),(D)$ and $(J)$, it is sufficient to prove our theorem only for the subgroups $(S)$ and $(D)$ and for the map $(J)$. Observing the matrix forms of $(S),(D),(K)$ and $\left(S^{\prime}\right)$ it is not difficult to obtain the explicit expression of the represention $T_{g}$ as follows:

$$
\left(T_{g} f\right)(x)=\left(\frac{1}{(x g)_{0}}\right)^{(n+1) / 2} f(\pi(\tilde{x} g)), \quad f \in L^{2}\left(\boldsymbol{R}^{n}, d x\right),
$$

where $\tilde{x} g=\left(x_{1}, \cdots, x_{n}, 1\right) \circ g$ and $(\tilde{x} g)_{i}$ is the $i$-th coordinate of the vector $\tilde{x} g$. By the definition of $U_{g}$ in (23), a short calculation shows that 


$$
\left(U_{g} h\right)(x)=\left(\frac{r}{\tilde{r}^{g}}\right)^{(n+1) / 2} h(\pi(\tilde{x} g)), \quad h \in L^{2}\left(\boldsymbol{R}^{n}, d \mathrm{Y}^{\prime}\right)
$$

where $r=\left(x_{1}^{2}+\cdots+x_{n}^{2}\right)^{1 / 2}$ and

$$
\tilde{r}^{g}=\left((\pi(\tilde{x} g))_{1}^{2}+\cdots+(\pi(\tilde{x} g))_{n}^{2}\right)^{1 / 2} .
$$

Set

$$
(\tilde{R} f)(\xi, p)=\left(\frac{\partial}{\partial p}\right)^{m} \check{f}(\xi, p), \quad \xi \in \boldsymbol{R}^{n}, p \in \boldsymbol{R}^{1}, m=(n-1) / 2 .
$$

Then we have

$$
R f(\eta)=(\tilde{R} f)(q,-1 / r), \quad \eta \in \boldsymbol{R}^{n},
$$

where $q=\left(q_{1}, \cdots, q_{n}\right)$ is the direction of $\eta$ and $r$ is the length of $\eta$, that is, $(q ; r)$ is the polar coordinate of $\eta$.

For simplicity we shall prove the theorem in the case $i=1$.

(S) Shift. For any element $f$ of $L^{2}\left(\boldsymbol{R}^{n}, d x\right)$,

$$
T_{S_{1}(t)} f\left(x_{1}, \cdots, x_{n}\right)=f\left(x_{1}+t, x_{2}, \cdots, x_{n}\right) d x
$$

and

$$
\begin{aligned}
\left(\tilde{R} T_{S_{1}(t)} f\right)(\xi, p) & =\left(\frac{\partial}{\partial p}\right)^{m} \int_{\langle\xi, x\rangle=p} f\left(x_{1}+t, x_{2}, \cdots, x_{n}\right) d x \\
& =\left(\frac{\partial}{\partial p}\right)^{m} \int_{\left\langle\xi_{1}\left(x_{1}+t\right)+\xi_{2} x_{2}+\cdots+\xi_{n} x_{n}\right\rangle=p} f(x) d x \\
& =\left(\frac{\partial}{\partial p}\right)^{m} \int_{\langle\xi, x\rangle=p-\xi_{1} t} f(x) d x \\
& =(\tilde{R} f)\left(\xi, p-\xi_{1} t\right) .
\end{aligned}
$$

Therefore, we have

$$
\begin{aligned}
\left(R T_{S_{i}(t)} f\right)(\eta) & =\left(\tilde{R} T_{S_{1}(t)} f\right)(q,-1 / r)=(\tilde{R} f)\left(q,(-1 / r)-q_{1} t\right) \\
& =(R f)\left(\eta^{g}\right)=U_{\hat{S}_{1}(t)}(R f)(\eta)
\end{aligned}
$$

where $\hat{A}$ means ${ }^{t} A^{-1}$ in the matrix form of $M(n)$ (see (40)),

$$
\eta^{g}=\left(\frac{\eta_{1}}{1+\eta_{1} t}, \frac{\eta_{2}}{1+\eta_{1} t}, \cdots, \frac{\eta_{n}}{1+\eta_{1} t}\right)
$$

and 


$$
\hat{S}_{1}(t)=\left(\begin{array}{cc|c}
1,0, \cdots, 0 & t \\
0,1,0, \cdot, 0 & 0 \\
\vdots & \cdots & \vdots \\
\vdots & , 1,0 & \vdots \\
0, \cdots, 0,1 & 0 \\
\hline 0,0, \cdots, 0 & 1
\end{array}\right)={ }^{t} S_{1}(t)^{-1}
$$

(D) For any element $f$ of $L^{2}\left(\boldsymbol{R}^{n}, d x\right)$, we have

$$
\left(T_{D_{1}(t)} f\right)(x)=e^{t / 2} f\left(e^{t} x_{1}, x_{2}, \cdots, x_{n}\right),
$$

and

$$
\left(\tilde{R} T_{D_{1}(t)} f\right)(\xi, p)=\left(\frac{\partial}{\partial p}\right)^{m} \int_{\langle\xi, x\rangle=p} e^{t / 2} f\left(e^{t} x_{1}, x_{2}, \cdots, x_{n}\right) d x
$$

$\left(x_{1}:=e^{t} x_{1}\right.$; the notation $y:=z$ means that we change the variable $z$ of the integral into $y$ )

$$
=\left(\frac{\partial}{\partial p}\right)^{m} e^{-t / 2} \int_{\left\langle\xi_{1} e^{-t} x_{1}+\xi_{2} x_{2}+\cdots+\xi_{n} x_{n}\right\rangle=p} f(x) d x=e^{-t / 2}(R f)\left(e^{-t} \xi_{1}, \xi_{2}, \cdots, \xi_{n}, p\right) .
$$

Therefore,

$$
\begin{aligned}
\left(R T_{D_{1}(t)}\right)(\eta) & =e^{-t / 2}(\tilde{R} f)\left(q_{1} e^{-t}, q_{2}, \cdots, q_{n},-1 / r\right) \\
& =e^{-t / 2}\left(\frac{1}{q_{1}^{2} e^{-2 t}+q_{2}^{2}+\cdots+q_{n}^{2}}\right)^{(n+1) / 4} R f\left(\eta^{g}\right) \\
& =e^{-t / 2}\left(\frac{r}{\left\|\eta^{g}\right\|}\right)^{(n+1) / 2} R f\left(\eta^{g}\right),
\end{aligned}
$$

where $\eta^{g}=\left(e^{-t} \eta_{1}, \eta_{2}, \cdots, \eta_{n}\right)$. Since

$$
\hat{D}_{1}(t)=\left(\begin{array}{c|c}
e^{-n t /(n+1)} & 0, \cdots, 0 \\
\hline 0, & \\
\vdots & e^{t /(n+1)} I_{n} \\
0 &
\end{array}\right.
$$

where $I_{n}$ in the $(n, n)$-identity matrix,

$$
\tilde{r}^{g}=e^{-t /(n+1)}\left\|\eta^{g}\right\|, \text { that is, } e^{-t / 2}\left(\frac{r}{\left\|\eta^{g}\right\|}\right)^{(n+1) / 2}=\left(\frac{r}{\tilde{r}^{g}}\right)^{(n+1) / 2}
$$

Thus we have

$$
R T_{D_{1}(t)}=U_{\hat{D}_{1}(t)} R
$$


(J) We note that

$$
\begin{array}{r}
\left(T_{J_{1}} f\right)(x)=\left(\frac{1}{x_{1}}\right)^{(n+1) / 2} f\left(-\frac{1}{x_{1}}, \frac{x_{2}}{x_{1}}, \cdots, \frac{x_{n}}{x_{1}}\right), \quad f \in L^{2}\left(\boldsymbol{R}^{n}, d x\right), \\
\left(U_{J_{1}} F\right)(\eta)=\left(\frac{\eta_{1}{ }^{2}+\cdots+\eta_{2}{ }^{2}}{1+\eta_{2}{ }^{2}+\cdots+\eta_{n}{ }^{2}}\right)^{(n+1) / 4} F\left(-\frac{1}{\eta_{1}}, \frac{\eta_{2}}{\eta_{1}}, \cdots, \frac{\eta_{n}}{\eta_{1}}\right), \\
F \in L^{2}\left(\boldsymbol{R}^{n}, d \zeta^{\prime}\right)
\end{array}
$$

and

$$
(R F)(\eta)=\frac{1}{\sqrt{2 \pi}} \int(-i \alpha)^{m} \tilde{F}\left(\alpha q_{1}, \cdots, \alpha q_{n}\right) e^{i \alpha / r} d \alpha
$$

where $\pi\left(\tilde{\eta} J_{1}\right)=\eta^{\prime}=\left(-1 /\left(r q_{1}\right), q_{2} / q_{1}, \cdots, q_{n} / q_{1}\right)$. Put $r^{\prime}=\left\|\eta^{\prime}\right\|$, then the polar coordinate of the vector $\eta^{\prime}$ is $\left(-1 /\left(r^{\prime} r q_{1}\right), q_{2} /\left(r^{\prime} q_{1}\right), \cdots, q_{n} /\left(r^{\prime} q_{1}\right) ; r^{\prime}\right)$. Therefore,

$$
\begin{aligned}
& \left(U_{J_{1}} R F\right)(\eta) \\
& \quad=\left(\frac{1}{q_{1} r^{\prime}}\right)^{m+1} \frac{1}{\sqrt{2 \pi}} \int_{-\infty}^{\infty}(-i \alpha)^{m} \tilde{F}\left(-\frac{\alpha}{r^{\prime} r q_{1}}, \frac{\alpha q_{2}}{r^{\prime} q_{1}}, \cdots, \frac{\alpha q_{n}}{r^{\prime} q_{1}}\right) e^{i \alpha / r^{\prime}} d \alpha \\
& \left(\alpha:=\alpha /\left(r^{\prime} q_{1}\right)\right) \\
& \quad=\left(\frac{1}{q_{1} r^{\prime}}\right)^{m+1} \frac{1}{\sqrt{2 \pi}} \int_{-\infty}^{\infty}\left(-i r^{\prime} q_{1} \alpha\right)^{m} \tilde{F}\left(-\frac{\alpha}{r}, \alpha q_{2}, \cdots, \alpha q_{n}\right) e^{i \alpha q_{1}}\left(r^{\prime} q_{1}\right) d \alpha \\
& \quad=\frac{1}{\sqrt{2 \pi}} \int_{-\infty}^{\infty}(-i \alpha)^{m} \tilde{F}\left(-\frac{\alpha}{r}, \alpha q_{2}, \cdots, \alpha q_{n}\right) e^{i \alpha q_{1}} d \alpha .
\end{aligned}
$$

On the other hand

$$
\begin{aligned}
\left(T_{J_{1}} F\right)^{\vee}(\xi, p) & \\
= & \left(\frac{1}{2 \pi}\right)^{m+1} \iint\left(\frac{1}{x_{1}}\right)^{m+1} F\left(-\frac{1}{x_{1}}, \frac{x_{2}}{x_{1}}, \cdots, \frac{x_{n}}{x_{1}}\right) \exp (i\langle x, \xi\rangle \alpha-i \alpha p) d x d \alpha \\
\left(x_{2}:=\right. & \left.x_{2} / x_{1}, \cdots, x_{n}:=x_{n} / x_{1}\right) \\
= & \left(\frac{1}{2 \pi}\right)^{m+1} \iint\left(x_{1}\right)^{m-1} F\left(-\frac{1}{x_{1}}, x_{2}, \cdots, x_{n}\right) \\
& \quad \times \exp \left[\left(i x_{1} \xi_{1}+x_{1}\left(x_{2} \xi_{2}+\cdots+x_{n} \xi_{n}\right)\right) \alpha-i \alpha p\right] d x d \alpha \\
(\alpha:= & \left.x_{1} \alpha\right) \\
= & \left(\frac{1}{2 \pi}\right)^{m+1} \iint\left(x_{1}\right)^{m-1} F\left(-\frac{1}{x_{1}}, x_{2}, \cdots, x_{n}\right) \\
& \quad \times \exp \left[i \alpha\left(\xi_{1}+x_{2} \xi_{2}+\cdots+x_{n} \xi_{n}-\frac{x}{x_{1}}\right)\right] d x d \alpha
\end{aligned}
$$


( $\mathscr{F}_{\hat{1}} F$ is the Fourier transform of $F$ with respect to the variables $x_{2}$, $\cdots, x_{n}$, and $\left.x_{1}:=-1 / x_{1}\right)$

$$
=\frac{1}{2 \pi} \iint\left(-\frac{1}{x_{1}}\right)^{m}\left(\mathscr{F}_{\hat{1}} F\right)\left(x_{1} ; \alpha \xi_{2}, \cdots, \alpha \xi_{n}\right) \exp \left[i \alpha\left(\xi_{1}+p x_{1}\right)\right] d x_{1} d \alpha .
$$

Hence we obtain

$$
\begin{aligned}
& \left(\tilde{R} T_{J_{1}} F\right)(\xi, p) \\
& =\frac{1}{2 \pi} \iint\left(i \alpha x_{1}\right)^{m}\left(-\frac{1}{x_{1}}\right)^{m}\left(\mathscr{F}_{\hat{1}} F\right)\left(x_{1} ; \alpha \xi_{2}, \cdots, \alpha \xi_{n}\right) \\
& \quad \times \exp \left[i \alpha\left(\xi_{1}+x_{1} p\right)\right] d x_{1} d \alpha \\
& =\frac{1}{\sqrt{2 \pi}} \int(-i \alpha)^{m} \tilde{F}\left(\alpha p, \alpha \xi_{2}, \cdots, \alpha \xi_{n}\right) e^{i \xi_{1} \alpha} d \alpha .
\end{aligned}
$$

Finaly we have

$$
\left(R T_{J_{1}} F\right)(\eta)=\frac{1}{\sqrt{2 \pi}} \int(-i \alpha)^{m} \tilde{F}\left(-\frac{\alpha}{r}, \alpha q_{2}, \cdots, \alpha q_{n}\right) e^{i \alpha q_{1}} d \alpha
$$

Thus comparing (75) with (74), we have

$$
R T_{J_{1}}=U_{J_{1}} R
$$

Noting that $\hat{J}_{1}=J_{1}$, we complete the proof of the main theorem.

\section{LITERATURES}

[1] Bochner, S.; Harmonic Analysis and the Theory of Probability, Univ. of Calif. Press (1960).

[2а] Гельфанд, И. М. и Виленкин, Н. Я.; Обобщенные Функции, вып. 4, Физматиз, М. (1961).

[2b] Гельфанд, И. М., Граев, М. И. и Виленкин, Н.Я.; Обобщенные Функции, вып. 5, Физматиз, М. (1962).

[3] Гихман, И. И. и Скороход, А. В.; Теоия Случайных Процессов, том. 1, Наука (1971).

[4] Helgason, S.; The Radon Transform on Euclidean Spaces, Compact Two-point Homogeneous Spaces and Grassman Manifolds, Acta Math. 113 (1965), 153-180.

[5a] Hida, T. Stationary Stochastic Processes, Princeton Univ. Press (1970).

[5b] - Complex White Noise and Infinite Dimensional Unitary Group, Lecture notes of Nagoya Univ. (1971).

[5c] —-; Brownian Motion, (in Japanese), Iwanimi (1975).

[5d] —-; Analysis of Brownian Functionals, Carleton Math. Lecture Notes No. 13 (1975).

[ 6 ] Hida, T. Kubo, I., Nomoto, H. and Yoshizawa, H.; On Projective Invariance of Brownian Motion, Publ. Res. Inst. Math. Sci. 4 (1968), 595-609.

[ 7 ] Hida T. and Ikeda, N.; Analysis on Hilbert Space with Reproducing Kernel 
Arising from Multiple Wiener Integral, 5th Berkeley Symp. (1965).

[8] Kôno, N.; On Hermite Polynomials, (in Japanese), Seminar on Probability vol. 27 (1967).

[9] Kubo, I.; Topics on Random Fields, (in Japanese), Seminar on Probability vol. 26 (1967).

[10a] Lévy, P.; Problèmes Concréts d'Analyse Fonctionnelles, Gauthier-Villars (1951).

[10b] — Processus Stochastiques et Mouvement Brownien, Gauthier-Villars (1965).

[11] Noda, A.; Gaussian Random Fields with Projective Invariance, Nagoya Math. J. 59 (1975), 65-76.

[12] Satô, H.; One-parameter Subgroups and a Lie Subgroup of an Infinite Dimensional Rotation Group, J. Math. Kyoto Univ. 11 (1971), 253-300.

[13] Umemura, Y.; Measures on Infinite Dimensional Vector Spaces, Publ. Res. Inst. Math. Sci. 1 (1966), 1-47.

[14] Wiener, N.; Nonlinear Problems in Random Theory, Technology Press of M.I.T. (1958).

[15] Yoshizawa, H.; Rotation Group of Hilbert Space and its Application to Brownian Motion, Proc. of the International Conference on Functional Analysis and Related Topics (1970), 414-425.

[16] Ченцов, Н.Н.; Многопараметрическое Броуновское Движение Леви и Обобщенный Белый шум, Теория вероятностей и ее применения, 2, (1957), 281-282.

Department of Mathematics

Nagoya University 